\title{
Supplementation with vitamin A early in life and subsequent risk of asthma
}

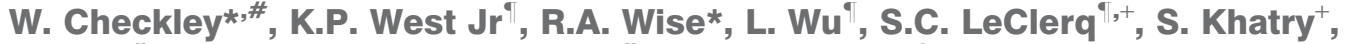 \\ J. Katz ${ }^{\#}$, P. Christian ${ }^{\star}$, J.M. Tielsch" and A. Sommer ${ }^{\S}$
}

ABSTRACT: Animal models suggest that vitamin A deficiency affects lung development adversely and promotes airway hyperresponsiveness, and may predispose to an increased risk of asthma. We examined the long-term effects of vitamin A supplementation early in life on later asthma risk.

In 2006-2008, we revisited participants from two cohorts in rural Nepal who were enrolled in randomised trials of vitamin A supplementation. The first cohort received vitamin A or placebo for $<16$ months during their pre-school years (1989-1991). The second cohort was born to mothers who received vitamin A, $\beta$-carotene or placebo before, during and after pregnancy (1994-1997). At follow-up, we asked about asthma symptoms and performed spirometry.

Out of 6,421 subjects eligible to participate, $5,430(85 \%)$ responded to our respiratory survey. Wheezing prevalence during the previous year was $4.8 \%$ in participants aged $9-13$ yrs and $6.6 \%$ in participants aged 14-23 yrs. We found no differences between the vitamin A supplemented and placebo groups from either trial in the prevalence of lifetime or current asthma and wheeze or in spirometric indices of obstruction ( $p \geqslant 0.12$ for all comparisons).

Vitamin A supplementation early in life was not associated with a decreased risk of asthma in an area with chronic vitamin A deficiency.

KEYWORDS: Asthma, longitudinal study, randomised clinical trial, vitamin A

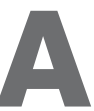
sthma is a chronic lung disease that is associated with airway inflammation, airflow limitation, bronchial hyperresponsiveness and symptoms of episodic wheeze and cough. Its prevalence has increased rapidly over the recent decades in both developed and developing countries and it has emerged as one of the most prevalent noncommunicable diseases worldwide. Asthma currently affects 300 million individuals worldwide, and is responsible for 180,000 deaths and 15 million disability-adjusted life-years lost each year [1]. The worldwide burden of disease caused by asthma is comparable to that of diabetes mellitus or cirrhosis [2, 3].

The cause of the increase in asthma prevalence is likely to be multifactorial. Rapid urbanisation and higher concentrations of outdoor air pollution in highly populated cities may have contributed to the rise $[4,5]$. A decrease in the incidence of infectious diseases may be associated with a shift in the cytokine balance and thus a greater risk of asthma, although this hypothesis remains unproven [6]. Dietary intake has also been implicated in the aetiology of asthma. In particular, a lower consumption of antioxidant vitamins may increase vulnerability to oxidative stress, which may in turn increase the risk or severity of asthma. Indeed, several observational studies have reported that lower levels of the antioxidant vitamins $\mathrm{C}$ and $\mathrm{E}$ are associated with a greater risk of asthma [7-12]. However, randomised controlled trials to examine the effect of supplementation with vitamins $C$ and E on asthma risk have been inconclusive [13-15].

The causal relationship between vitamin A deficiency and asthma risk is less well characterised. Vitamin A is an antioxidant that plays a key role in lung development and regeneration of epithelial lung tissue $[16,17]$. We recently reported that offspring born to mothers who received vitamin A before, during and after pregnancy as part of a randomised trial had better lung function than did those whose mothers received placebo [18]. Animal experiments have identified that vitamin A deficiency is associated with airway hyperresponsiveness [19] and that supplementation with all-trans retinoic acid, an active metabolic intermediate of vitamin A, reverses airway hyperresponsiveness associated with vitamin A deficiency [20]. While multiple observational studies [21-25] have found that vitamin A deficiency is associated

\section{AFFILIATIONS}

*Division of Pulmonary and Critical

Care, School of Medicine,

\#Program in Global Disease

Epidemiology and Control,

"Center for Human Nutrition, Dept of International Health, and,

${ }^{\S}$ Dept of Epidemiology, Bloomberg School of Public Health, Johns

Hopkins University, Baltimore, MD, USA.

${ }^{+}$The Nepal Nutrition Intervention Project Sarlahi (NNIPS), National Society for the Prevention of Blindness, Kathmandu, Nepal.

\section{CORRESPONDENCE}

W. Checkley

Division of Pulmonary and Critical Care

Dept of Medicine

Johns Hopkins University

Baltimore

MD 21212

USA

E-mail: wcheckl@jhmi.edu

Received:

Jan 142011

Accepted after revision:

April 262011

First published online:

June 232011 
with a higher risk of asthma, there are no randomised trials to support a causal relationship.

We undertook the present study to determine the effects of supplementation early in life with pre-formed vitamin A on subsequent asthma risk in an area with chronic vitamin A deficiency. To achieve this aim, we revisited participants from two cohorts of placebo-controlled vitamin A supplementation trials in rural Nepal. The first cohort received vitamin A or placebo during their pre-school years [26]. The second cohort was born to mothers who received vitamin $A, \beta$-carotene or placebo before, during and after pregnancy [27]. Our hypothesis was that vitamin A deficiency early in life, which is known to affect pulmonary development adversely and promote airway hyperresponsiveness, may predispose to an increased risk of asthma during childhood and through young adulthood.

\section{METHODS}

\section{Study setting}

We conducted our study in the Sarlahi District, located in the densely populated, low-lying southern plains of southern Nepal, known as the Terai. The Terai is an area of chronic under-nutrition and vitamin A deficiency, where earlier surveys have repeatedly observed childhood xerophthalmia to be a public health problem [28-30], attributable to early weaning from the breast and a traditional rice-based diet low in animal, vegetable and fruit sources of vitamin A.

\section{Original trials}

The first trial, Nepal Nutritional Intervention Project-Sarlahi (NNIPS)-1, was a double-blind, placebo-controlled, cluster randomised trial, conducted between September 1989 and May 1991, to evaluate the effect of 4-monthly, high-potency vitamin A supplementation on pre-school child mortality [26]. We mapped and addressed households in 29 contiguous village development committees (VDCs), each with nine wards (our unit of randomisation), and conducted a baseline enumeration at which time we enrolled children aged $\leqslant 60$ months into the trial. On each subsequent 4-monthly visit, infants born during the previous interval were also enrolled. The ages of children were updated at each visit. On each occasion, children aged $\geqslant 12$ months were given, by trained field staff, an oil-based supplement containing placebo or 200,000 IU of vitamin A $(60,000 \mu \mathrm{g}$ retinol equivalents (RE)), according to their ward assignment. Infants aged 1-11 months of age were offered a half-dose (placebo or 30,000 $\mu \mathrm{g}$ RE) and newborns aged $<1$ month a quarter-dose (placebo or $15,000 \mu \mathrm{g} R E$ ). During a 16-month period we enrolled, dosed and followed a total of 28,630 children. Vitamin A was found to reduce the risk of childhood death by $30 \%$ after the fourth visit [26]. Thereafter, all children aged 6-60 months received vitamin A supplementation throughout the last 8 months of the trial while the youngest infants remained in the trial. As a result, the supplementation intervals among children were variable. The majority of children received four doses over 16 months $(>70 \%)$, whereas a smaller proportion received only one dose of vitamin A $(<5 \%)$. We concurrently enrolled a subsample of 6,617 children into a more detailed substudy from 40 (15\%) of the 261 wards, which were randomly sampled within geographical quadrants of the study area (10 from each quadrant). Out of these children, 6,462 (98\%) were alive at the end of the trial.
The second trial (NNIPS-2) was a double-blind, placebocontrolled, cluster randomised trial of married females, conducted between April 1994 and September 1997, to evaluate the effect of weekly supplementation with vitamin A or $\beta$-carotene on mortality from all causes related to pregnancy [27]. We invited all eligible females from 30 VDCs to participate in this trial (total 270 wards, of which 261 were from NNIPS-1). The unit of randomisation was the ward, and each ward within a VDC was block-randomised to one of three treatments. We enrolled and continuously supplemented 44,646 females each week with an oral gelatin capsule containing 7,000 $\mu \mathrm{g}$ RE of provitamin A, $42 \mathrm{mg}$ of $\beta$-carotene, or placebo. Supplementation with vitamin A or $\beta$-carotene reduced pregnancy-related mortality by $44 \%$ versus placebo [27]. Neither supplement had an effect on infant mortality [31]. We concurrently enrolled pregnant females and their liveborn infants from a subsample of three contiguous VDCs (27 out of 270 wards) into a more detailed clinical and biochemical assessment protocol. 2,055 children were born alive to mothers in the subsample who completed the pregnancy-to-post partum dosing protocols, of whom 1,894 (92\%) were alive at the end of the trial. All children aged 6-60 months received biannual vitamin A supplementation through a national programme.

\section{Follow-up study}

In 2006, we revisited the households of NNIPS-1 participants and offspring of NNIPS-2 mothers and invited them to participate in a follow-up study. We used a household list derived from the original trials to generate an updated list of children who were eligible to participate in the follow-up study between October 2006 and March 2008. At follow-up, we conducted a survey in which we asked participants about respiratory symptoms (see online supplementary material). We selected the questions for our survey from recommended questionnaires [32, 33]. We also asked participants to undergo spirometry using a portable spirometer (SpiroPro; Jaeger, Hoechberg, Germany). Each participant performed spirometry in the sitting position with a noseclip, until three acceptable and reproducible manoeuvres out of a maximum of eight were obtained, in accordance with standard guidelines [34]. Technicians reviewed all of the flow-volume curves performed each day with a supervisor. Flow-volume curves were transmitted weekly to the investigators in the USA for additional review. We performed direct supervision and in-person review of all flowvolume curves with each technician approximately every 3 months. This study was approved by the institutional review boards of the Johns Hopkins University (Baltimore, MD, USA) and the Institute of Medicine, Tribhuvan University (Kathmandu, Nepal).

\section{Biostatistical methods}

We aimed to determine whether there were differences in the prevalence of asthma or in measures of airflow limitation [35] at follow-up according to supplement assignment. We employed an intent-to-treat analysis and fit regression models using generalised estimating equations with a compound symmetry correlation matrix [36] to account for clustering by ward. We calculated simple ward-level summaries for all outcomes and compared the ward-level means of forced expiratory volume (FEV1) to forced vital capacity (FVC) ratio, peak expiratory flow (PEF), maximum mid-expiratory flow (MMEF) and forced expiratory flow at 75\% of FVC (FEF75\%) 
by supplement assignment using standard techniques [37]. Socioeconomic indicators, age or sex did not confound the effects of supplement assignment on outcome estimates (data not shown). Multivariable adjustment by height did not confound the effect of supplement assignment on spirometric indices of obstruction (see online supplementary material). We used R (www.r-project.org) and SAS (Cary, NC, USA) for statistical analysis.

\section{RESULTS}

\section{Characteristics of the NNIPS-1 study population}

The status of participants from the original NNIPS-1 subsample, aged $14-23$ yrs at follow-up, is summarised in figure 1. Out of 6,462 children who were alive at the end of the trial, 1,405 (21.7\%) were no longer living in the study area and $303(4.7 \%)$ had died. No information was available for 20 $(0.4 \%)$ participants. 4,523 were eligible to participate and 3,879 ( $86 \%$ of those eligible) responded to our respiratory survey. 3,345 attempted spirometry (74\% of those eligible), and 3,075 performed spirometry according to standard criteria. We did not find differences between study groups in the proportion of participants who died $(p=0.45)$, who moved out of the study area $(p=0.58)$, who did not complete the respiratory survey $(p=0.11)$ or who refused to participate in the study $(p=0.69)$. Participants who completed the respiratory survey were more likely to be of higher caste $(p=0.01)$, to live in households that

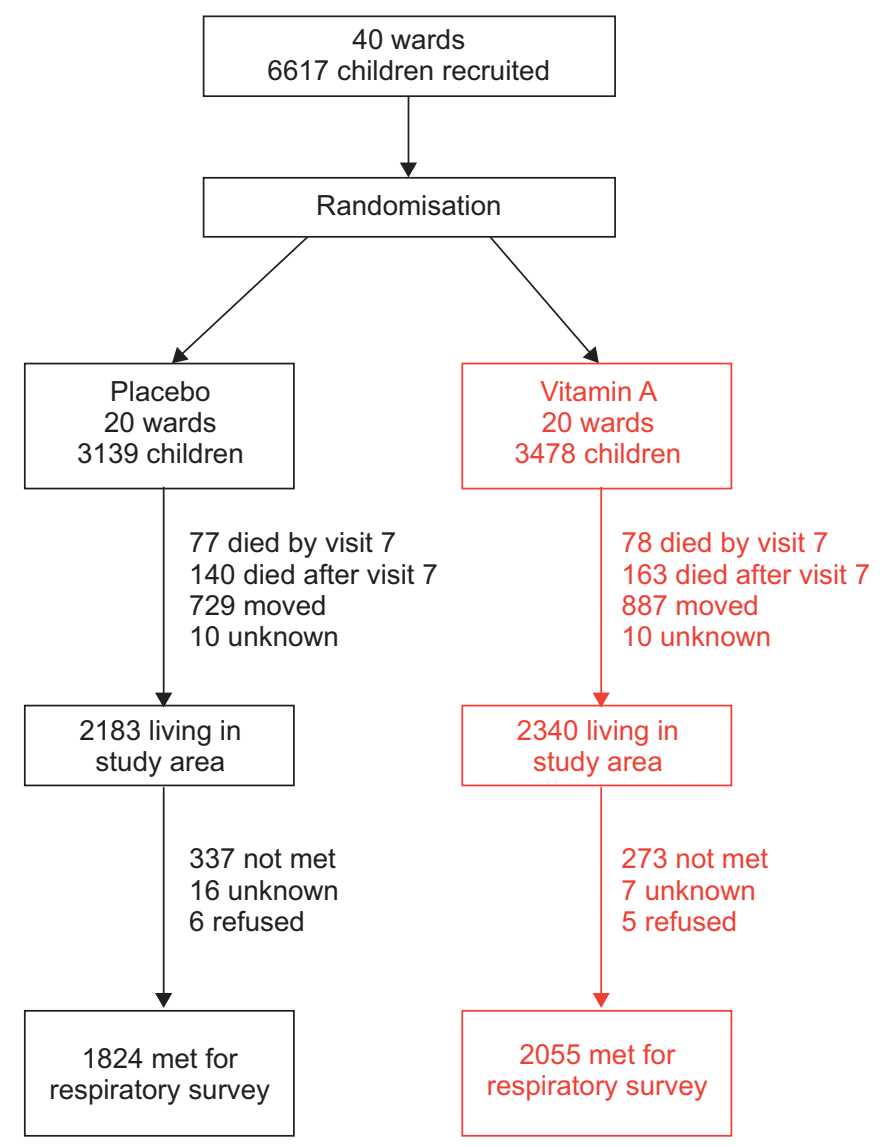

FIGURE 1. Eligibility for census and enrolment of study participants aged 14$23 \mathrm{yrs}$ from the original 6,617 children of a well-defined subsample. Nepal Nutritional Intervention Project-Sarlahi (NNIPS)-1 follow-up study, 2006-2008. owned land $(p<0.001)$, to own livestock $(p<0.001)$ and to have a father who was a farmer $(\mathrm{p}<0.001)$ than the remaining 2,738 participants from the original substudy. We did not find important differences in demographic or socioeconomic status between study groups at follow-up (table 1).

\section{Characteristics of the NNIPS-2 study population}

The status of participants from the NNIPS-2 original subsample, aged 9-13 yrs at follow-up, is summarised in figure 2. Out of 1,894 children who were alive at the end of the trial, 118 $(6.2 \%)$ were no longer living in the study area and $110(5.8 \%)$ had died. No information was available for $8(0.4 \%)$ participants. 1,658 children were eligible to participate and 1,551 ( $93.5 \%$ of those eligible) responded to our respiratory survey. 1,371 children attempted spirometry ( $83 \%$ of those eligible), and 1,322 performed spirometry according to standard criteria. We did not find differences between study groups in the proportion of children who died before the close-out of the trial on September 30, $1997(p=0.09)$, who died after September $30,1997(p=0.62)$, who moved out of the study area $(p=0.78)$, who did not complete the respiratory survey $(p=0.55)$ or who refused to participate in the study $(p=0.40)$. Children who completed the respiratory survey were more likely to be of higher caste $(p=0.04)$, to live in households that owned land $(\mathrm{p}<0.001)$, to own livestock $(\mathrm{p}<0.001)$ and to have a father who was a farmer $(\mathrm{p}<0.001)$ than the remaining 504 participants from the original substudy. We did not find important differences in demographic or socioeconomic status between study groups at follow-up (table 2).

\section{Prevalence of asthma}

There was no difference in lifetime or current self-reported asthma in adolescent to adult participants who received vitamin A supplementation or placebo in their pre-school years (table 1) or in children born to mothers who received vitamin A supplementation, $\beta$-carotene or placebo before, during and after pregnancy (table 2) at follow-up. Among those who reported ever having asthma in either cohort, only 30\% (26 out of 88 ) reported being actually diagnosed by a physician or health worker, suggesting low access to medical personnel in the region. The low proportion of participants who reported having asthma in both cohorts vis-à-vis the higher prevalence of wheezing suggests that there is a large subset of individuals who have under-recognised, and possibly under-treated, asthma.

\section{Prevalence of wheezing}

The overall prevalence of wheezing in the previous year was $6.6 \%(254$ out of 3,852$)$ in participants aged $14-23$ yrs and $4.8 \%$ (73 out of 1,522) in participants aged 9-13 yrs at follow-up. There was no difference in the prevalence of either lifetime or current wheezing in adolescent and adult participants who received vitamin A supplementation or placebo in their preschool years (table 1), or in children who were born to mothers who received vitamin A supplementation, $\beta$-carotene or placebo before, during and after pregnancy (table 2). Of those who reported wheezing in the previous year, 50.2\% (164 out of 327) also reported that they had at least one wheezing attack accompanied by shortness of breath and $45.0 \%$ (147 out of 327) were awakening at night by wheezing. We did not find any differences between supplement assignment groups in 
TABLE 1 Sample size, demographics, socioeconomic indicators, and prevalence of asthma, wheezing and other respiratory symptoms in 3,879 children who responded to the respiratory survey by supplement assignment group. Nepal Nutritional Intervention Project-Sarlahi (NNIPS)-1 follow-up study, 2006-2008

\begin{tabular}{|c|c|c|c|}
\hline & \multicolumn{2}{|c|}{ Supplement assignment after birth } & \multirow[t]{2}{*}{ p-value } \\
\hline & Placebo & Vitamin A & \\
\hline \multicolumn{4}{|l|}{ Sample size } \\
\hline Wards $n$ & 20 & 20 & \\
\hline Children n & 1824 & 2055 & \\
\hline Age yrs & $18.7 \pm 2.1$ & $18.7 \pm 2.1$ & 0.73 \\
\hline Age yrs (mean of ward means) & $18.6 \pm 0.3$ & $18.7 \pm 0.3$ & 0.71 \\
\hline Sex males & 57 & 59 & 0.29 \\
\hline Sex males (mean of ward proportions) & 58 & 59 & 0.40 \\
\hline \multicolumn{4}{|l|}{ Ward summaries of SES } \\
\hline House without roof or with a thatch roof & 40 & 36 & 0.74 \\
\hline House without walls or thatch-made walls & 81 & 82 & 0.83 \\
\hline Maternal literacy & 10 & 9 & 0.74 \\
\hline Father is farmer & 70 & 66 & 0.34 \\
\hline \multicolumn{4}{|l|}{ Ward summaries of asthma prevalence } \\
\hline Lifetime asthma (self-report) & 1.6 & 1.9 & 0.29 \\
\hline Physician diagnosis of asthma & 0.8 & 0.6 & 0.75 \\
\hline Age at onset of asthma yrs & $11.2 \pm 6.0$ & $11.3 \pm 6.0$ & 0.85 \\
\hline Current asthma (self-report) & 0.6 & 0.6 & 0.98 \\
\hline \multicolumn{4}{|l|}{ Ward summaries of wheezing prevalence } \\
\hline Lifetime wheeze & 11.3 & 12.2 & 0.24 \\
\hline$>1$ night $\cdot$ week $^{-1}$ & 1.9 & 1.9 & 0.63 \\
\hline \multicolumn{4}{|l|}{ Ward summaries of cough and phlegm prevalence } \\
\hline Persistent cough (coughs more than normal) & 7.3 & 7.0 & 0.83 \\
\hline Chronic cough (3 months of consecutive cough in a 2-yr period) & 2.6 & 2.2 & 0.70 \\
\hline Time with cough yrs & $2.8 \pm 2.3$ & $4.2 \pm 2.6$ & 0.08 \\
\hline Persistent phlegm (produces phlegm more than normal) & 12.4 & 10.3 & 0.29 \\
\hline Chronic phlegm ( 3 months of consecutive phlegm production in a 2-yr period) & 3.7 & 3.3 & 0.50 \\
\hline Chronic productive cough & 1.2 & 1.0 & 0.60 \\
\hline Dry cough at night in the past year & 6.2 & 5.8 & 0.83 \\
\hline Time with phlegm yrs & $2.1 \pm 1.2$ & $2.3 \pm 1.3$ & 0.37 \\
\hline Chest illnesses after colds & 23.1 & 24.9 & 0.49 \\
\hline Phlegm episodes with chest illness $n$ & $4.1 \pm 5.7$ & $2.3 \pm 1.2$ & 0.86 \\
\hline
\end{tabular}

either cohort (tables 1 and 2) with respect to the number of attacks per year, wheezing accompanied by shortness of breath or wheezing-related night-time awakenings.

\section{Prevalence of cough and phlegm}

The prevalence of chronic productive and nonproductive cough in the two cohorts was nine per 1,000 participants (47 


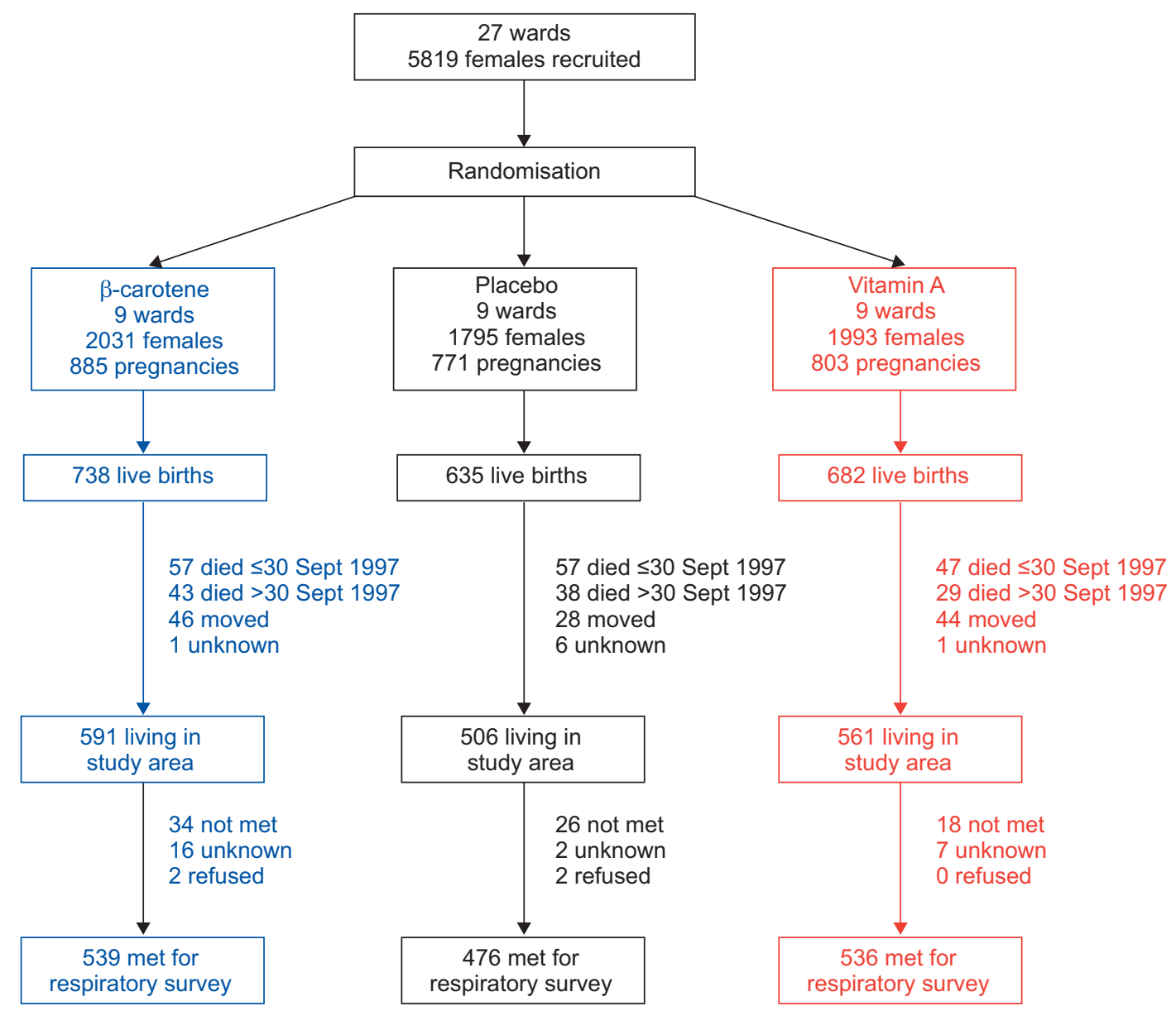

FIGURE 2. Eligibility for census and enrolment of study participants aged $9-13$ yrs from the original 2,055 live births of a well-defined subsample. Nepal Nutritional Intervention Project-Sarlahi (NNIPS)-2 follow-up study, 2006-2008

out of 5,427) and 13 per 1,000 participants (70 out of 5,427), respectively. We did not find differences in cough or phlegm symptoms between study groups in either cohort (tables 1 and 2). Stratified by sex and cohort, females aged 14-23 yrs had a similar prevalence of chronic productive cough as males (10 versus 12 per 1,000 participants, respectively; $\mathrm{p}=0.64)$. Girls aged 9-13 yrs had a similar prevalence of chronic productive cough as boys of a similar age (four versus one per 1,000 participants, respectively; $\mathrm{p}=0.37$ ).

\section{Spirometric indices of obstruction}

The distributions of FEV1/FVC by sex and cohort are shown in figure 3. We did not find any differences in FEV1/FVC, PEF, FEF75\% or MMEF by supplement assignment in NNIPS-1 participants (table 3). Despite a 46-mL increase in FEV1 and FVC among the offspring of females who received vitamin A versus placebo [18], there were no differences in FEV1/FVC, PEF, FEF75\% or MMEF between study groups (table 4).

\section{DISCUSSION}

This study aimed to investigate the effect of vitamin A supplementation early in life on the subsequent risk of asthma in two cohorts of participants enrolled at different developmental periods in placebo-controlled vitamin A supplementation trials.
In the first trial, a large dose of vitamin A or placebo was given to study participants every 4 months for 16 months beginning when they were aged $\leqslant 5$ yrs, after which all children received prophylactic vitamin A until age 6 yrs. In the second trial, vitamin A, $\beta$-carotene, or placebo was given weekly to mothers of study participants before, during and after pregnancy for $\sim 3$ yrs. After 1994, all pre-school-aged children were subsequently exposed to a high-coverage semi-annual vitamin A supplementation as part of a national programme [38]. The results of our study indicate that vitamin A supplementation early in life did not affect the prevalence of asthma or measures of airways obstruction in participants aged 9-23 yrs.

There are several reasons why vitamin A deficiency, despite our findings, may be associated with a higher prevalence of asthma. Increased asthma might be expected in vitamin A-deficient individuals because vitamin $\mathrm{A}$ is necessary for proper lung epithelial cell differentiation and lung development [39-41]. Therefore, it would be reasonable to speculate that early lung development abnormalities may be implicated in the early origins of asthma. Animal studies have shown that vitamin A deficiency promotes airway hyperresponsiveness and alters the mechanical properties of the lung parenchyma. Vitamin A deficiency is also associated with a reduced abundance and function of muscarinic $\mathrm{M}_{2}$ receptors, which may help explain the 
TABLE 2 Sample size, demographics, socioeconomic indicators and prevalence of asthma, wheezing, and other respiratory symptoms in 1,551 children who responded to the respiratory survey by maternal supplement assignment group. Nepal Nutritional Intervention Project-Sarlahi (NNIPS)-2 follow-up study, 2006-2008

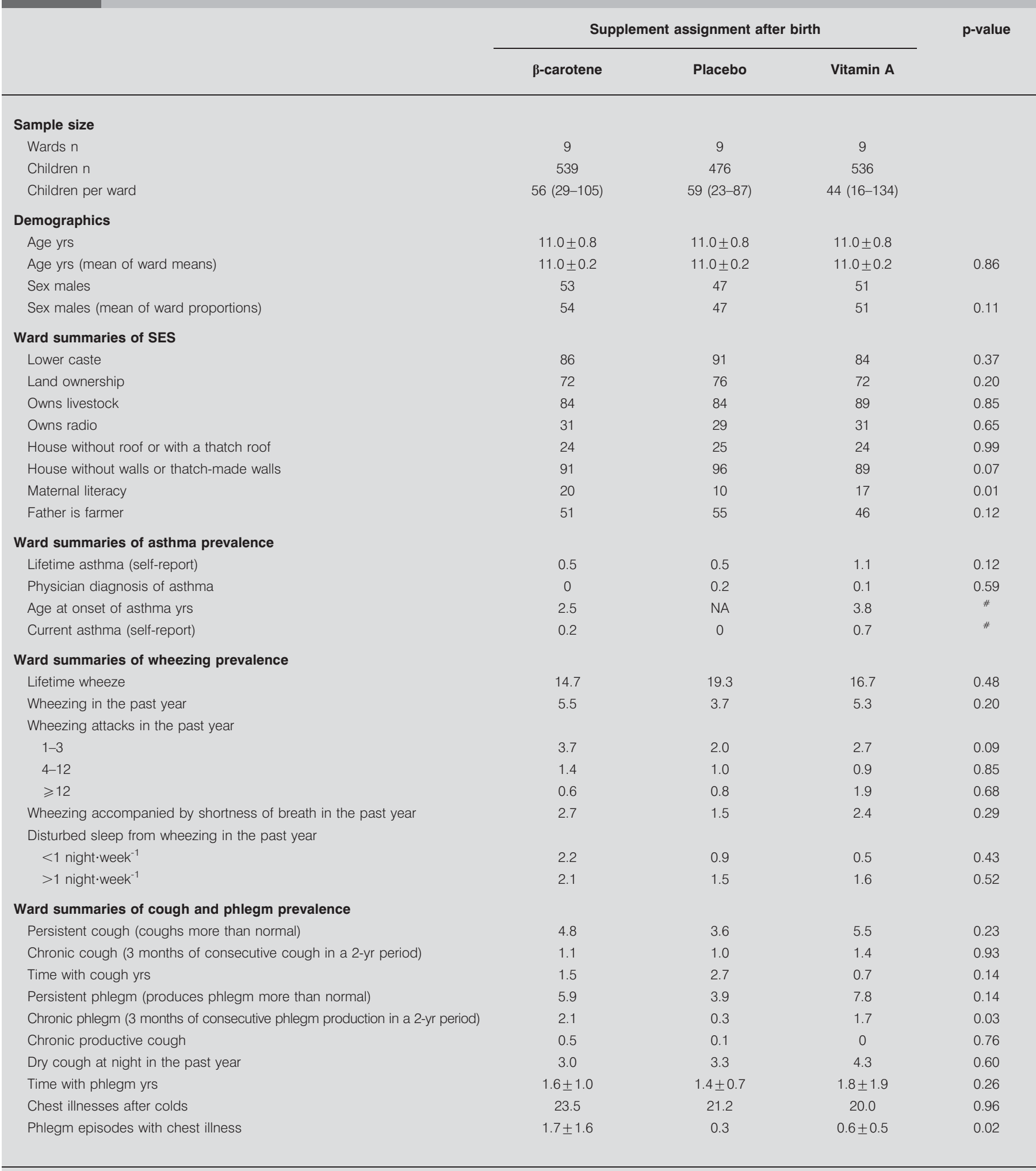

Data are presented as median (range), mean \pm SD or $\%$, unless otherwise stated. SES: socioeconomic status. NA: not available. ${ }^{\#}$ : could not estimate because of small sample size. 


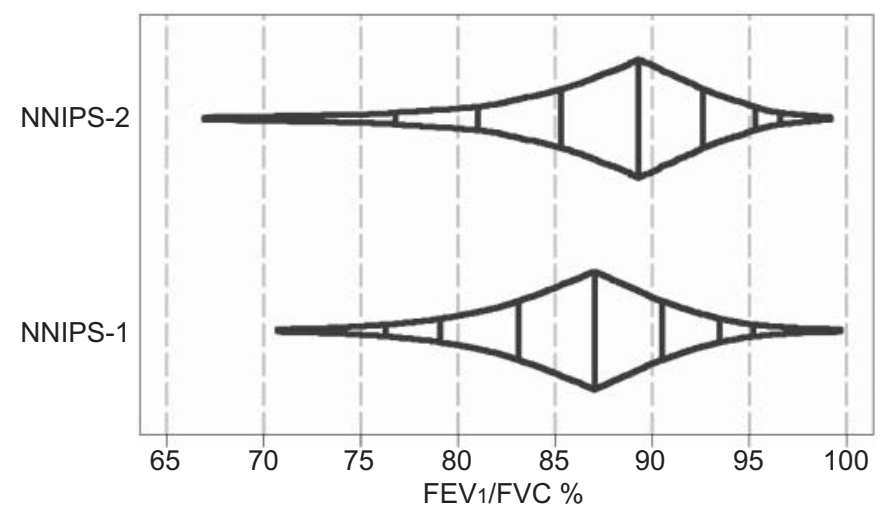

FIGURE 3. Box-percentile plot of forced expiratory volume in $1 \mathrm{~s}(\mathrm{FEV} 1)$ to forced vital capacity (FVC) ratio in study participants aged 9-23 yrs. Nepal Nutritional Intervention Project-Sarlahi (NNIPS)-1 and -2 follow-up study, 20062008. The box-percentile plot uses width to encode information about the distribution of the data over the entire range of data values. diminished ability to limit cholinergic-mediated bronchoconstriction [19]. While supplementation with retinoic acid reverses the vitamin A deficiency-induced airway hyperresponsiveness and the decrease in muscarinic $\mathrm{M}_{2}$ receptors [20], it does not appear to reverse alterations in parenchymal mechanics and structure resulting from vitamin A deficiency [42].

Our findings, however, conflict with available epidemiological evidence from observational studies on the association between vitamin A and asthma [20-24]. Thus, we need to consider reasons for why we may have not seen an effect of vitamin A supplementation on asthma. One possibility is that asthma may alter the metabolism of vitamin A. Factors implicated in asthma exacerbations, such as pulmonary infections or airway inflammation, have been shown to decrease serum retinol by increasing cellular demand or urinary excretion of retinol [23, 43-45]. Thus, inflammation causing reduction in serum retinol may explain why observational studies have found an association between asthma and vitamin A [20-24] and would explain why

TABLE 3 Spirometric indicators of obstructive airways disease in 3,075 children by supplement assignment group. Nepal Nutritional Intervention Project-Sarlahi (NNIPS)-1 follow-up study, 2006-2008

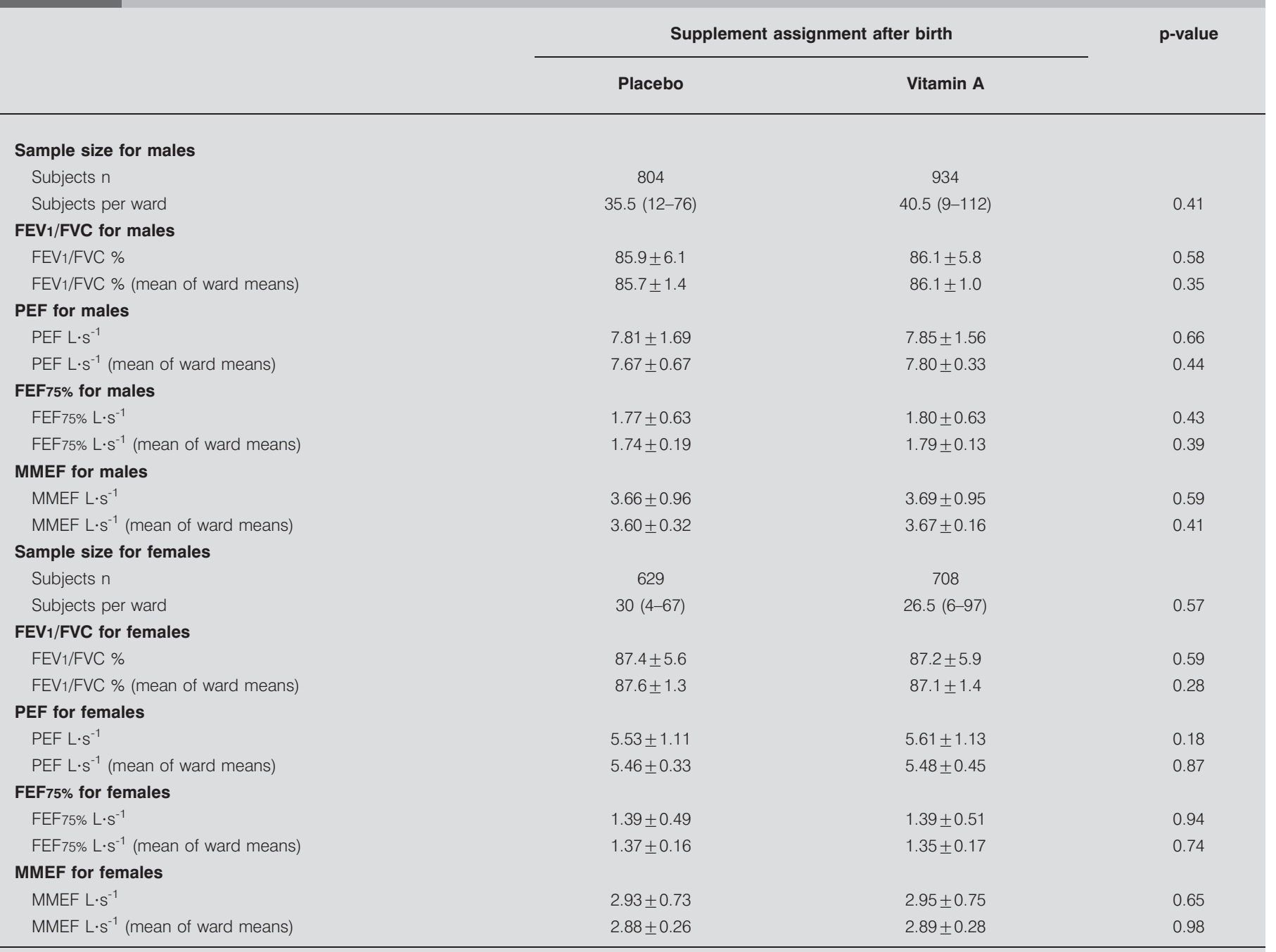

Data are presented as median (range) or mean \pm SD, unless otherwise stated. FEV1: forced expiratory volume in 1s; FVC: forced vital capacity; PEF: peak expiratory flow; FEF75\%: forced expiratory flow at $75 \%$ of FVC; MMEF: maximum mid-expiratory flow. 
TABLE 4 Spirometric indicators of obstructive airways disease in 1,322 children by maternal supplement assignment group. Nepal Nutritional Intervention Project-Sarlahi (NNIPS)-2 follow-up study, Nepal, 2006-2008

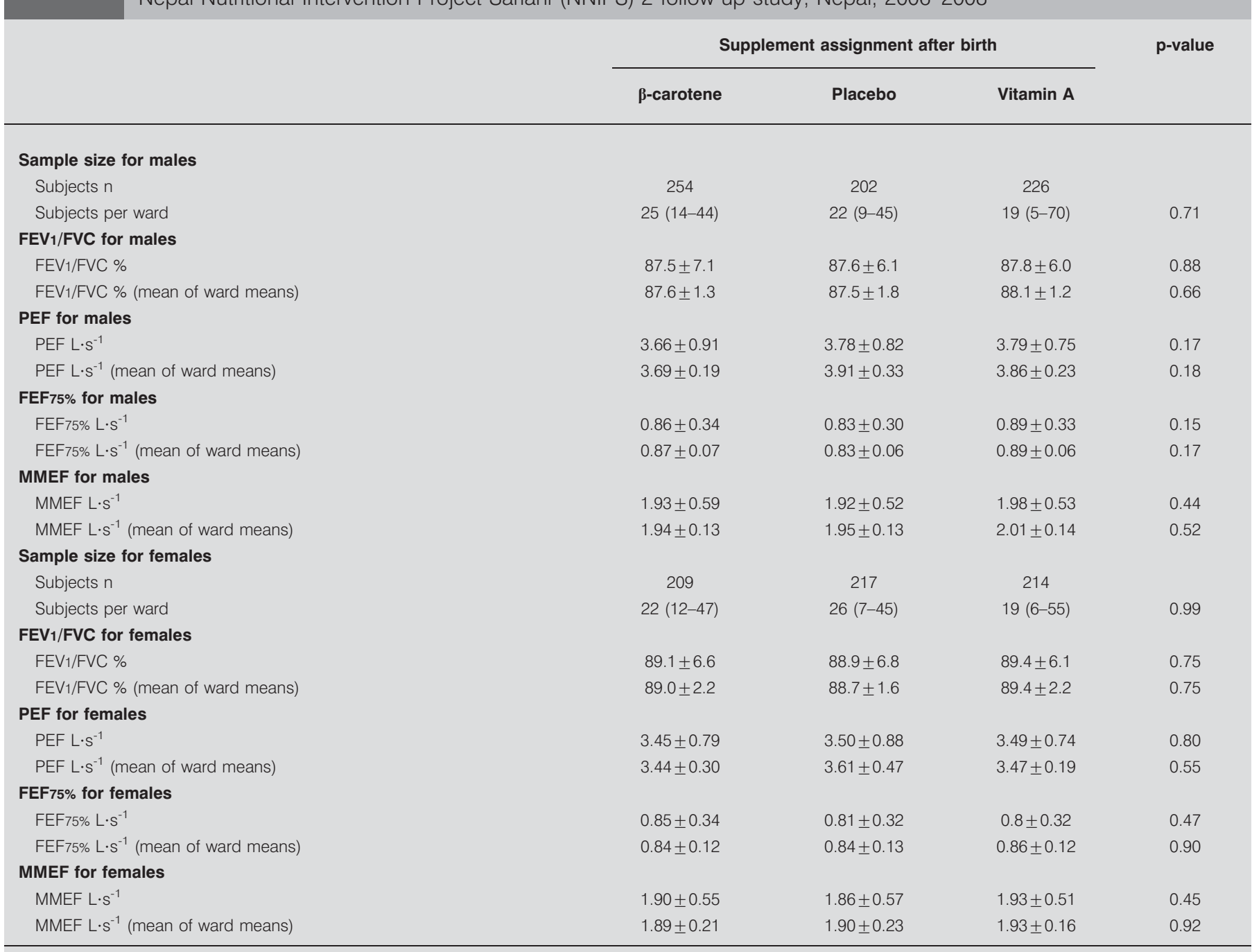

Data are presented as median (range) or mean \pm SD, unless otherwise stated. FEV1: forced expiratory volume in $1 \mathrm{~s}$; FVC: forced vital capacity; PEF: peak expiratory flow FEF75\%: forced expiratory flow at $75 \%$ of FVC; MMEF: maximum mid-expiratory flow.

vitamin A supplementation did not reduce the risk of asthma in either of our trials. Another possibility is that incomplete followup of the original trial participants because of loss to follow-up or death may have affected our findings. Our follow-up study included $59 \%$ of the original NNIPS- 1 subsample and $75 \%$ of the original NNIPS-2 subsample. However, we did not find differences in participants' characteristics according to supplement assignment at follow-up. Differential mortality between supplement allocation groups, as observed in NNIPS-1 but not in NNIPS-2, could lead to a survivor bias where children who died might have been at greater risk of having asthma; however, the number of children who died in the NNIPS-1 trial was too small (11-16 deaths per 1,000 child-yrs) [25] to result in an important survivor bias. A third possibility is that the duration of supplementation was relatively short: a maximum of 16 months in NNIPS-1 trial participants, and during and shortly after pregnancy for the offspring of NNIPS-2 trial participants.
Future studies may need to consider longer periods of vitamin A supplementation in the critical years of lung development $[46,47]$ to study the long-term effects of asthma risk. A fourth possibility is that implementation of a national vitamin A supplementation programme in pre-school-aged children may have masked the effects of early childhood supplements provided during our trials. A fifth possibility is that early vitamin A supplementation affects lung growth later in life [18] by increasing both FEV1 and FVC roughly equally, suggesting that lung size and airway calibre were symmetrically influenced by vitamin A supplementation. Finally, compliance with supplementation could have affected our results; however, this is unlikely because compliance in both trials was high. Specifically, compliance with vitamin A supplementation in the NNIPS-1 children was $>90 \%$, and $>75 \%$ of the NNIPS- 2 pregnant females received at least half of their eligible doses of vitamin $\mathrm{A}$ or $\beta$-carotene (i.e., more than half of a dietary allowance). 
One limitation of this study is that we were not able to evaluate airway hyperresponsiveness or reversibility in this study. Airway hyperresponsiveness, evaluated by means of a methacholine challenge, would have provided additional data to support the relationship between vitamin A and asthma, but it would have been less feasible for us to perform given that our study was designed to visit participants at their homes. Furthermore, our study was not designed to have medically trained personnel at all times in the field in case of any serious adverse events. Data regarding nutrition from birth to the time of follow-up and retinol levels at the time of follow-up were not available and would have helped us to better understand the relationship between asthma and vitamin A.

In summary, early vitamin A supplementation did not increase asthma risk. While vitamin A deficiency early in life affects pulmonary development adversely, it was not associated with an increased prevalence of asthma in an area with chronic vitamin A deficiency.

\section{SUPPORT STATEMENT}

This work was carried out by the Center for Human Nutrition, Department of International Health of the Johns Hopkins Bloomberg School of Public Health (Baltimore, MD, USA) and the Division of Pulmonary and Critical Care of the Johns Hopkins University, School of Medicine, The Johns Hopkins University (Baltimore) through the Nepal Nutrition Intervention Project-Sarlahi in collaboration with the National Society for Comprehensive Eye Care (Kathmandu, Nepal). This study was funded by the Bill and Melinda Gates Foundation (Seattle, WA, USA; grant number 614) and the Sight and Life Research Institute (Baltimore). The original maternal vitamin A or $\beta$-carotene supplementation trial (1994-1997) was conducted under the Vitamin A for Health Cooperative Agreement number HRN-A-00-97-00015-00 between the Johns Hopkins University and the Office of Health, Infectious Diseases and Nutrition of the US Agency for International Development (Washington, DC, USA), with additional support from Task Force Sight and Life (Basel, Switzerland). W. Checkley was further supported by a Clinician Scientist Award from the Johns Hopkins University and a Pathway to Independence Award from the National Heart, Lung and Blood Institutes (Baltimore; award number K99HL096955). The sponsors had no role in the study design, writing of the report or decision to submit the paper for publication.

\section{STATEMENT OF INTEREST}

None declared.

\section{ACKNOWLEDGEMENTS}

We especially thank the late S. Ram Shrestha for his dedicated contributions to the field study.

\section{REFERENCES}

1 Braman SS. The global burden of asthma. Chest 2006; 130: 4-12.

2 Masoli M, Fabian D, Holt S, et al. The global burden of asthma: executive summary of the GINA Dissemination Committee Report. Allergy 2004; 59: 469-478.

3 Bousquet J. The public health implications of asthma. Bull World Health Organ 2005; 83: 548-554.

4 Yemaneberhan H, Bekele Z, Venn A, et al. Prevalence of wheeze and asthma and relation to atopy in urban and rural Ethiopia. Lancet 1997; 350: 85-90.

5 Committee of the environmental and occupational health assembly of the American Thoracic Society. Health effects of outdoor air pollution: part I of II. Am J Respir Crit Care Med 1996; 153: 3-50.

6 Busse W, Lemanske RF. Asthma. N Eng J Med 2001; 344: 350-362.
7 Schwartz J, Weiss ST. Dietary factors and their relation to respiratory symptoms. The Second National Health and Nutrition Examination Survey. Am J Epidemiol 1990; 132: 67-76.

8 Troisi RJ, Willett WC, Weiss ST, et al. A prospective study of diet and adult-onset asthma. Am J Respir Crit Care Med 1995; 151: 1401-1408.

9 Misso NL, Brooks-Wildhaber J, Ray S, et al. Plasma concentrations of dietary and nondietary antioxidants are low in severe asthma. Eur Respir J 2005; 26: 257-264.

10 Kelly FJ, Mudway I, Blomberg A, et al. Altered lung antioxidant status in patients with mild asthma. Lancet 1999; 354: 482-483.

11 Bodner C, Godden D, Brown K, et al. Antioxidant intake and adult-onset wheeze: a case-control study. Aberdeen WHEASE Study Group. Eur Respir J 1999; 13: 22-30.

12 Schünemann HJ, Grant BJ, Freudenheim JL, et al. The relation of serum levels of antioxidant vitamins $\mathrm{C}$ and $\mathrm{E}$, retinol and carotenoids with pulmonary function in the general population. Am J Respir Crit Care Med 2001; 163: 1246-1255.

13 Fogarty A, Lewis SA, Scrivener SL, et al. Oral magnesium and vitamin $C$ supplements in asthma: a parallel group randomized placebo-controlled trial. Clin Exp Allergy 2003; 33: 1355-1359.

14 Pearson PJ, Lewis SA, Britton J, et al. Vitamin E supplements in asthma: a parallel group randomised placebo controlled trial. Thorax 2004; 59: 652-656.

15 Romieu I, Sienra-Monge JJ, Ramírez-Aguilar M, et al. Antioxidant supplementation and lung functions among children with asthma exposed to high levels of air pollutants. Am J Respir Crit Care Med 2002; 166: 703-709.

16 Wilson JG, Roth CB, Warkany J. An analysis of the syndrome of malformations induced by maternal vitamin A deficiency. Effects of restoration of vitamin A at various times during gestation. Am J Anat 1953; 92: 189-217.

17 Chytil F. Retinoids in lung development. FASEB J 1996; 10: 986-992.

18 Checkley W, West KP Jr, Wise RA, et al. Effects of maternal vitamin A supplementation on lung function in preadolescent offspring: a follow-up study of a randomized, double-blinded, placebo-controlled trial cohort in rural Nepal. N Engl J Med 2010; 362: 1784-1794

19 McGowan SE, Smith J, Holmes AJ, et al. Vitamin A deficiency promotes bronchial hyperreactivity in rats by altering muscarinic $\mathrm{M}_{2}$ receptor function. Am J Physiol Lung Cell Mol Physiol 2002; 282: L1031-L1039.

20 McGowan SE, Holmes AJ, Smith J. Retinoic acid reverses the airway hyperresponsiveness but not the parenchymal defect that is associated with vitamin A deficiency. Am J Physiol Lung Cell Mol Physiol 2004; 286: L437-L444.

21 Arora P, Kumar V, Batra S. Vitamin A status in children with asthma. Pediatr Allergy Immunol 2002; 13: 223-226.

22 Riccioni G, Bucciarelli T, Mancini B, et al. Plasma lycopene and antioxidant vitamins in asthma: the PLAVA study. J Asthma 2007; 44: 429-432.

23 Mizuno Y, Furusho T, Yoshida A, et al. Serum vitamin A concentrations in asthmatic children in Japan. Pediatr Int 2006; 48: 261-264.

24 Morabia A, Menkes MJ, Comstock GW, et al. Serum retinol and airway obstruction. Am J Epidemiol 1990; 132: 77-82.

25 Allen S, Britton JR, Leonardi-Bee JA. Association between antioxidant vitamins and asthma outcome measures: systematic review and meta-analysis. Thorax 2009; 64: 610-619.

26 West KP Jr, Pokhrel RP, Katz J, et al. Efficacy of vitamin A in reducing preschool child mortality in Nepal. Lancet 1991;338: 67-71.

27 West KP Jr, Katz J, Khatry SK, et al. Double blind, cluster randomised trial of low dose supplementation with vitamin A or $\beta$-carotene on mortality related to pregnancy in Nepal. BMJ 1999; 318: 570-575.

28 Brilliant LB, Pokhrel RP, Grasset NC, et al. Epidemiology of blindness in Nepal. Bull World Health Organ 1985; 63: 375-386. 
29 Upadhyay MP, Gurung BJ, Pillai KK, et al. Xerophthalmia among Nepalese children. Am J Epidemiol 1985; 121: 71-77.

30 Khatry SK, West KP Jr, Katz J, et al. Epidemiology of xerophthalmia in Nepal. A pattern of household poverty, childhood illness, and mortality. The Sarlahi Study Group. Arch Ophthalmol 1995; 113: 425-429.

31 Katz J, West KP Jr, Khatry SK, et al. Maternal low-dose vitamin A or $\beta$-carotene supplementation has no effect on fetal loss and early infant mortality: a randomized cluster trial in Nepal. Am J Clin Nutr 2000; 71: 1570-1576.

32 Asher MI, Keil U, Anderson HR, et al. International Study of Asthma and Allergies in Childhood (ISAAC): rationale and methods. Eur Respir J 1995; 8: 483-491.

33 Ferris BG. Epidemiology standardization project (American Thoracic Society). Recommended respiratory disease questionnaires for use with adults and children in epidemiological research. Am Rev Respir Dis 1978; 118: 7-52.

34 American Thoracic Society. Standardization of spirometry (1994 update). Am J Respir Crit Care Med 1995; 152: 1107-1136.

35 Sears MR, Greene JM, Willan AR, et al. A longitudinal, populationbased, cohort study of childhood asthma followed to adulthood. N Engl J Med 2003; 349: 1414-1422.

36 Liang KY, Zeger SL. Regression analysis for correlated data. Annu Rev Public Health 1993; 14: 43-68.

37 Hayes RJ, Moulton LH. Cluster Randomized Trials. London, Chapman \& Hall/CRC Press, 2008; pp. 149-231.
38 Nepal Micronutrient Status Survey 1998. Kathmandu, Ministry of Health, Child Health Division, His Majesty's Government of Nepal/NewERA, Micronutrient Initiative/UNICEF Nepal/World Health Organization; pp. 54-66.

39 Wolbach SB, Howe PR. Tissue changes following deprivation of fat-soluble vitamin A. J Exp Med 1925; 42: 753-777.

40 Sharma HS, Mishra UK. Postnatal distribution of vitamin A in liver, lung, heart and brain of the rat in relation to maternal vitamin A status. Biol Neonate 1986; 50: 345-350.

41 Zachman RD. Role of vitamin A in lung development. J Nutr 1995; 125: 1634S-1638S.

42 McGowan SE, Takle EJ, Holmes AJ. Vitamin A deficiency alters the pulmonary parenchymal elastic modulus and elastic fiber concentration in rats. Respir Res 2005; 6: 77.

43 Filteau SM, Morris S, Abbott RA, et al. Influence of morbidity on serum retinol of children in a community-based study in northern Ghana. Am J Clin Nutr 1993; 58: 192-197.

44 Morabia A, Sorenson A, Kumanyika SK, et al. Vitamin A, cigarette smoking, and airway obstruction. Am Rev Respir Dis 1989; 140: 1312-1316.

45 Riccioni G, Barbara M, Bucciarelli $\mathrm{T}$, et al. Antioxidant vitamin supplementation in asthma. Ann Clin Lab Sci 2007; 37: 96-101.

46 Galambos C, DeMello DE. Regulation of alveologenesis: clinical implications of impaired growth. Pathology 2008; 40: 124-140.

47 Burri PH. Structural aspects of postnatal lung development alveolar formation and growth. Biol Neonate 2006; 89: 313-322. 